\title{
An Overview of Research Areas in Hybrid Control
}

\author{
John Lygeros
}

\begin{abstract}
Hybrid systems have been an active area of research for a number of years. Recently a consensus is beginning to emerge among researchers about theoretical and applied problems related to control of hybrid systems that are both important and tractable. In this overview paper we survey recent research advances and highlight some of the open problems.
\end{abstract}

\section{INTRODUCTION}

The term hybrid systems is used in the literature to refer to systems that feature an interaction between diverse types of dynamics. Most heavily studied in recent years are hybrid systems that involve the interaction between continuous dynamics and discrete dynamics. The study of this class of systems has to a large extent been motivated by applications to embedded systems and control. Embedded systems by definition involve the interaction of digital devices with a predominantly analog environment. In addition, much of the design complexity of embedded systems comes from the fact that they have to meet specifications such as hard realtime constraints, scheduling constraints, etc. that involve a mixture of discrete and continuous requirements. Therefore, both the model and the specifications of embedded systems can naturally be expressed in the context of hybrid systems. Motivated by the observation that embedded systems often also have to deal with an uncertain and potentially adverserial environment, researchers have in recent years extended their study of hybrid systems beyond continuous and discrete dynamics, to include probabilistic terms. This has led to the more general class of stochastic hybrid systems.

Control problems have been at the forefront of hybrid systems research from the very beginning. The reason is that many important applications with prominent hybrid dynamics come from the area of embedded control. For example, hybrid control has played an important role in applications to avionics, automated highways, automotive control, air traffic management, industrial process control, and manufacturing and robotics; advances in many of these application areas will be surveyed in the remaining papers of this tutorial.

The control problems that have arisen in these applications differ, first of all, in the way in which they treat uncertainty. Generally, the problems can be grouped into three classes:

1) Deterministic. Here it is assumed that there is no uncertainty; control inputs are the only class of inputs considered.

Work carried out in the framework of the HYCON Network of Excellence, contract number FP6-IST-511368.

John Lygeros is with the Faculty of Electrical Engineering, University of Patras, 26500 Rio, Patras, Greece, Iygeros@ee.upatras.gr
2) Non-deterministic. In this case inputs are grouped into two classes, control and disturbance. The design of a controller for regulating the control inputs assumes that disturbance inputs are adverserial. Likewise, the requirements are stated as worst case: the controller should be such that the specifications are met for all possible actions of the disturbance. From a control perspective, problems in this class are typically framed in the context of robust control, or game theory.

3) Stochastic. Again, both control and disturbance inputs are considered. The difference with the nondeterministic case is that a probability distribution is assumed for the disturbance inputs. This extra information can be exploited by the controller and also allows one to formulate finer requirements. For example, it may not be necessary to meet the specifications for all disturbances, as long as the probability of meeting them is high enough.

In addition, the control problems studied in the literature differ in the specifications they try to meet. Generally, according to the specification the problems can also be grouped into three classes:

1) Stabilization. Here the problem is to select the continuous inputs and/or the timing and destinations of discrete switches to make sure that the system remains close to an equilibrium point, limit cycle, or other invariant set. Many variants of this problem have been studied in the literature. They differ in the type of control inputs considered (discrete, continuous, or both) and the type of stability specification (stabilization, asymptotic or exponential stabilization, practical stabilization, etc.). Even more variants have been considered in the case of stochastic hybrid systems (stability in distribution, moment stability, almost sure asymptotic stability, etc.).

2) Optimal control. Here the problem is to steer the hybrid system using continuous and/or discrete controls in a way that minimizes a certain cost function. Again, different variants have been considered, depending on whether discrete and/or continuous inputs are available, whether cost is accumulated along continuous evolution and/or during discrete transitions, whether the time horizon over which the optimization is carried out is finite or infinite, etc.

3) Language specifications. Control problems of great interest can also be formulated by imposing the requirement that the trajectories of the closed-loop system are all contained in a set of desirable trajectories. 
Typical requirements of this type arise from reachability considerations, either of the safety type (along all trajectories the state of the system should remain in a "good" region of the state space), or of the liveness type (the state of the system should eventually reach a "good" region of the state space along all trajectories). Starting with these simple requirements, progressively more and more complex specifications can be formulated: the state should visit a given set of states infinitely often, given two sets of states, if the state visits one infinitely often it should also visit the other infinitely often, etc. These specifications are all related to the "language" generated by the closedloop system and have been to a large extent motivated by analogous problems formulated for discrete systems based on temporal logic.

In this paper we provide an introduction to the problems addressed in all these areas. In Section III we formulate a number of hybrid stabilization problems, state the main approaches to solving these problems, and provide references to publications where more details can be found. In Sections IV and V we do the same with optimal control problems and language specification problems, respectively. To be able to clearly state the different control problems of interest, we start by introducing a simple hybrid system model (Section II). We stress that this hybrid model is meant to be used only for illustration purposes. It is not the model used in any of the references, nor does it claim to be a general model for controlled hybrid systems.

\section{A Simple Hybrid Control Model}

Hybrid control problems have been formulated for both continuous- and discrete-time systems. As usual, continuoustime problems present more technical difficulties. In this section we introduce a model suitable for formulating continuous-time control problems for deterministic hybrid systems. We also discuss briefly the simplifications that arise if discrete-time systems are considered and the complications involved in extending the model to stochastic systems.

\section{A. Syntax: Non-deterministic systems}

Since we are interested in hybrid dynamics, the dynamical systems we consider involve both a continuous state (denoted by $x$ ) and a discrete state (denoted by $q$ ). To allow us to capture the different types of uncertainties discussed above, we also assume that the evolution of the state is influenced by two different kinds of inputs: controls and disturbances. We assume that inputs of each kind can be either discrete or continuous, and we use $v$ to denote discrete controls, $u$ to denote continuous controls, $\delta$ to denote discrete disturbances, and $d$ to denote continuous disturbances.

The dynamics of the state are determined through four functions: a vector field $f$ that determines the continuous evolution, a reset map $r$ that determines the outcome of the discrete transitions, a "guard" set that determines when discrete transitions can take place, and a "domain" set Dom that determines when continuous evolution is possible. The following definition formalizes the details.

Definition 1 (Hybrid game automaton): A hybrid game automaton (HGA) characterizes the evolution of

- discrete state variables $q \in Q$ and continuous state variables $x \in X$

- discrete control inputs $v \in \Upsilon$ and continuous control inputs $u \in U$ and

- discrete disturbance inputs $\delta \in \Delta$ and continuous disturbance inputs $d \in D$

by means of four functions

- a vector field $f: Q \times X \times U \times D \rightarrow X$,

- a domain set Dom : $Q \times \Upsilon \times \Delta \rightarrow 2^{X}$,

- guard sets $G: Q \times Q \times \Upsilon \times \Delta \rightarrow 2^{X}$, and

- a reset function $r: Q \times Q \times X \times U \times D \rightarrow X$.

As usual, $2^{X}$ stands for the set of all subsets (power set) of $X$; in other words, Dom and $G$ are set-valued maps. For simplicity, we assume that $X=\mathbb{R}^{n}, U \subseteq \mathbb{R}^{m}$, and $D \subseteq \mathbb{R}^{p}$ for integers $n, m$, and $p$. A similar definition can also be formulated for discrete-time hybrid systems, simply by considering $f$ as a transition function rather than as a vector field. To avoid pathological situations (lack of solutions, deadlock, chattering, etc.) one needs to introduce technical assumptions on the model components. Typically, these include continuity assumptions on $f$, compactness assumptions on $U$ and $D$, and convexity assumptions on $\bigcup_{u \in U} f(q, x, u, d)$ and $\bigcup_{d \in D} f(q, x, u, d)$, needed to ensure existence of continuous solutions. Additional assumptions are often imposed to prevent deadlock, a situation where it is not possible to proceed by continuous evolution or by discrete transition. A typical assumption to prevent this situation is that the $\operatorname{set} \operatorname{Dom}(q, v, \delta)$ is open and if $x \notin$ $\operatorname{Dom}(q, v, \delta)$ then $x \in \bigcup_{q^{\prime} \in Q} G\left(q, q^{\prime}, v, \delta\right)$. Finally, in many publications assumptions are introduced to prevent what is called the Zeno phenomenon, a situation where the solution of the system takes an infinite number of discrete transitions in a finite amount of time. The Zeno phenomenon can prove particularly problematic for hybrid control problems, since it may be exploited either by the control or by the disturbance variables. For example, a controller may appear to meet a safety specification by forcing all trajectories of the system to be Zeno. This situation is undesirable in practice, since the specifications are met not because of successful controller design but because of modeling over-abstraction. In addition, Zeno controllers require infinitely fast switching and cannot be implemented in practice. For these reasons, the Zeno phenomenon is usually forbidden by direct assumptions. In some cases, structural assumptions are introduced on the model to prevent Zeno solutions (e.g., by enforcing a lower bound on the time between discrete transitions or the time to traverse each discrete state cycle).

Many of the assumptions discussed here can be relaxed, replaced by other variants, or dropped altogether; for example, if we consider relaxed controls in optimal control problems, convexity and compactness assumptions are typically not needed. For discrete-time hybrid systems, most of these 
assumptions are unnecessary. For example, deadlock and the Zeno phenomenon are typically not issues for discrete-time systems.

\section{B. Semantics: Solutions or runs}

To formally define the solutions of this class of hybrid systems, we recall the following notion from [1].

Definition 2 (Hybrid time set): A hybrid time set $\tau=$ $\left\{I_{i}\right\}_{i=0}^{N}$ is a finite or infinite sequence of intervals of the real line, such that

- for all $i<N, I_{i}=\left[\tau_{i}, \tau_{i}^{\prime}\right]$;

- if $N<\infty$, then either $I_{N}=\left[\tau_{N}, \tau_{N}^{\prime}\right]$, or $I_{N}=$ $\left[\tau_{N}, \tau_{N}^{\prime}\right)$, possibly with $\tau_{N}^{\prime}=\infty$;

- for all $i, \tau_{i} \leq \tau_{i}^{\prime}=\tau_{i+1}$.

Since the dynamical systems considered here are time invariant, without loss of generality we can assume that $\tau_{0}=0$. It easy to see that, although more complicated than the usual time sets (the real numbers for continuous-time systems or the integers for discrete-time systems), hybrid time sets are reasonably well-behaved mathematical objects. For example, each hybrid time set is totally ordered, whereas the set of all hybrid time sets is partially ordered. One can therefore naturally define prefixes and suffixes of a hybrid time set, maximal elements of a collection of hybrid time sets, etc. For discrete-time hybrid systems, the introduction of hybrid time sets is unnecessary, since the set of integers or natural numbers can typically be used.

Roughly speaking, the solution of an HGA (often called a "run" or an "execution") is defined over a hybrid time set $\tau$ and involves a sequence of intervals of continuous evolution followed by discrete transitions. Starting at some initial state $\left(q_{0}, x_{0}\right)$ the continuous state moves along the solution of the differential equation $\dot{x}=f\left(q_{0}, x, u, d\right)$ as long as it does not leave the set $\operatorname{Dom}\left(q_{0}, v, \delta\right)$. The discrete state remains constant throughout this time. If at some point $x$ reaches a set $G\left(q_{0}, q^{\prime}, v, \delta\right)$ for some $q^{\prime} \in Q$, a discrete transition can take place. The first interval of $\tau$ ends and the second one begins with a new state $\left(q^{\prime}, x^{\prime}\right)$ where $x^{\prime}$ is determined by the reset map $r$. The process is then repeated. Notice that considerable freedom is allowed when defining the solution in this "declarative" way: in addition to the effect of the input variables, there may also be a choice between evolving continuously or taking a discrete transition (if the continuous state is in both the domain set and a guard set) or between multiple discrete transitions (if the continuous state is in many guard sets at the same time).

The following concept helps to formalize the above discussion.

Definition 3 (Hybrid trajectory): Given a set of variables, $a$, that take values in a set $A$, a hybrid trajectory over this set of variables is a pair $(\tau, a)$ where $\tau=\left\{I_{i}\right\}_{i=0}^{N}$ is a hybrid time set and $a=\left\{a_{i}(\cdot)\right\}_{i=0}^{N}$ is a sequence of functions $a_{i}(\cdot)$ : $I_{i} \rightarrow A$.

The solutions of the HGA can now be defined as hybrid trajectories over its state and input variables.

Definition 4 (Run): A run of an HGA is a hybrid trajectory $(\tau, q, x, v, u, \delta, d)$ over its state and input variables that satisfies the following conditions:

- Discrete evolution: for $i<N$,

1) $x_{i}\left(\tau_{i}^{\prime}\right) \in G\left(q_{i}\left(\tau_{i}^{\prime}\right), q_{i+1}\left(\tau_{i+1}\right), v_{i}\left(\tau_{i}^{\prime}\right), \delta_{i}\left(\tau_{i}^{\prime}\right)\right)$.

2) $x_{i+1}\left(\tau_{i+1}\right)=$

$$
r\left(q_{i}\left(\tau_{i}^{\prime}\right), q_{i+1}\left(\tau_{i+1}\right), x_{i}\left(\tau_{i}^{\prime}\right), u_{i}\left(\tau_{i}^{\prime}\right), d_{i}\left(\tau_{i}^{\prime}\right)\right) .
$$

- Continuous evolution: for all $i$ with $\tau_{i}<\tau_{i}^{\prime}$

1) $u_{i}(\cdot)$ and $d_{i}(\cdot)$ are measurable functions.

2) $q_{i}(t)=q_{i}\left(\tau_{i}\right)$ for all $t \in I_{i}$.

3) $x_{i}(\cdot)$ is a solution of the differential equation

$$
\dot{x}_{i}(t)=f\left(q_{i}(t), x_{i}(t), u_{i}(t), d_{i}(t)\right)
$$

over the interval $I_{i}$ starting at $x_{i}\left(\tau_{i}\right)$.

4) $x_{i}(t) \in \operatorname{Dom}\left(q_{i}(t), v_{i}(t), \delta_{i}(t)\right)$ for all $t \in\left[\tau_{i}, \tau_{i}^{\prime}\right)$.

\section{Classification of control action}

The preceding model allows control and disturbance inputs to influence the evolution of the system in a number of ways. In particular, control and disturbance can

1) Steer the continuous evolution through the effect of $u$ and $d$ on the vector field $f$.

2) Force discrete transitions to take place through the effect of $v$ and $\delta$ on the domain Dom.

3) Affect the discrete state reached after a discrete transition through the effect of $v$ and $\delta$ on the guards $G$.

4) Affect the continuous state reached after a discrete transition through the effect of $u$ and $d$ on the reset function $r$.

Notice that the model implicitly restricts the influence of the discrete inputs $v$ and $\delta$ to the timing and discrete destination of discrete transitions and the influence of the continuous inputs $u$ and $d$ to continuous evolution and the continuous destination of discrete transitions. At this level of generality all inputs could, in fact, be allowed to influence all aspects of the evolution of the system. Caution should be taken, however, when doing this, since experience suggests that it tends to severely complicate the technicalities associated with the definition of runs, ensuring that runs exist for all inputs, preventing chattering strategies, etc. Experience also suggests that this type of mixing of discrete and continuous inputs is rarely needed in practice.

Another issue that arises is the type of controllers one allows for selecting the control inputs $u$ and $v$. The most common control strategies considered in the hybrid systems literature are, of course, static feedback strategies. In this case the controller can be thought of as a map (in general set valued) of the form $g: Q \times X \rightarrow 2^{\Upsilon \times U}$. For controllers of this type, the runs of the closed-loop system can easily be defined as runs, $(\tau, q, x, v, u, \delta, d)$, of the uncontrolled system such that for all $I_{i} \in \tau$ and all $t \in I_{i},\left(v_{i}(t), u_{i}(t)\right) \in$ $g\left(q_{i}(t), x_{i}(t)\right)$.

It turns out that for certain kinds of control problems (for example, reachability problems) one can restrict attention to feedback controllers without loss of generality. For other problems, however, one may be forced to consider more general classes of controllers: dynamic feedback controllers that incorporate observers for output feedback problems, 
controllers that involve non-anticipative strategies for gaming problems, piecewise constant controllers to prevent chattering, etc. Even for these types of controllers, it is usually intuitively clear what one means by the runs of the closedloop system. However, unlike feedback controllers, a formal definition would require one to formulate the problem in a compositional hybrid systems framework and formally define the closed-loop system as the composition of a plant and a controller automaton.

\section{Stochastic hybrid systems}

Stochastic terms can enter hybrid dynamics in a number of different places:

1) Continuous evolution may be governed by stochastic differential equations.

2) Discrete transitions may take place spontaneously, at a given, possibly state-dependent, rate (as they do for example in discrete Markov chains). Some authors also consider forced transitions, which take place whenever the continuous state tries to leave a given set (the equivalent of the Dom set introduced above).

3) The destination of discrete transitions may be given by a probability kernel.

As for deterministic and non-deterministic systems, one can also consider controls that influence the same places: for example, controls that steer continuous evolution through controlled diffusions, influence the rate at which discrete transitions take place, determine the boundaries at which they are forced, or influence the probability distribution that determines the destination of discrete transitions. Clearly, all these alternatives allow for the formulation of countless variants of control problems.

\section{Stabilization OF HybRid Systems}

The problem of stabilizing hybrid systems is designing controllers such that the runs of the closed-loop system remain close and possibly converge to a given invariant set. An invariant set is a set of states with the property that runs starting in the set remain in the set forever. More formally, $W \subseteq Q \times X$ is an invariant set if for all $(\hat{q}, \hat{x}) \in W$ and all runs $(\tau, q, x, v, u, \delta, d)$ starting at $(\hat{q}, \hat{x})$,

$$
\left(q_{i}(t), x_{i}(t)\right) \in W, \forall I_{i} \in \tau, \forall t \in I_{i} .
$$

The most common invariant sets are those associated with equilibria, points $\hat{x} \in X$ that are preserved under both discrete and continuous evolution.

The definitions of stability can naturally be extended to hybrid systems by defining a metric on the hybrid state space. An easy way to do this is to consider the Euclidean metric on the continuous space and the discrete metric on the discrete space $\left(d_{D}\left(q, q^{\prime}\right)=0\right.$ if $q=q^{\prime}$ and $d_{D}\left(q, q^{\prime}\right)=1$ if $\left.q \neq q^{\prime}\right)$ and define the hybrid metric by

$$
d_{H}\left((q, x),\left(q^{\prime}, x^{\prime}\right)\right)=d_{D}\left(q, q^{\prime}\right)+\left\|x-x^{\prime}\right\| .
$$

The metric notation can be extended to sets in the usual way. Equipped with this metric, the standard stability definitions (Lyapunov stability, asymptotic stability, exponential stability, practical stability, etc.) naturally extend from the continuous to the hybrid domain. For example, an invariant set, $W$, is called stable if for all $\epsilon>0$ there exists $\epsilon^{\prime}>0$ such that for all $(q, x) \in Q \times X$ with $d_{H}((q, x), W)<\epsilon^{\prime}$ and all runs $(\tau, q, x, v, u, \delta, d)$ starting at $(q, x)$,

$$
d_{H}\left(\left(q_{i}(t), x_{i}(t)\right), W\right)<\epsilon, \quad \forall I_{i} \in \tau, \forall t \in I_{i} .
$$

Stability of hybrid systems has been extensively studied in recent years (see the overview papers $[2,3]$ ). By comparison, the work on stabilization problems is relatively sparse. A family of stabilization schemes assumes that the continuous dynamics are given, for example, stabilizing controllers have been designed for each $f(q, \cdot, \cdot, \cdot)$. Procedures are then defined for determining the switching times (or at least constraints on the switching times) to ensure that the closedloop system is stable, asymptotically stable, or practically stable [4-7]. Stronger results are possible for special classes of systems, such as planar systems [8]. For non-deterministic systems, in [9] an approach to the practical exponential stabilization of a class of hybrid systems with disturbances is presented. For a brief overview of stabilization problems for stochastic hybrid systems the reader is referred to [10].

\section{Optimal Control of Hybrid Systems}

In optimal control problems it is typically assumed that a cost is assigned to the different runs of the hybrid system by means of a cost function. The objective of the controller is then to minimize this cost among all possible runs by selecting the values of the control variables appropriately. Typically, the cost function assigns a cost to both continuous evolution and discrete transitions. For example, for the cost assigned to a run $(\tau, q, x, v, u, \delta, d)$ with $\tau=\left\{I_{i}\right\}_{i=0}^{N}$, the cost function may have the form

$$
\begin{gathered}
\sum_{i=0}^{N}\left[\int_{\tau_{i}}^{\tau_{i}^{\prime}} l\left(q_{i}(t), x_{i}(t), u_{i}(t), d_{i}(t)\right) d t\right. \\
\quad+g\left(q_{i}\left(\tau_{i}^{\prime}\right), x_{i}\left(\tau_{i}^{\prime}\right), q_{i+1}\left(\tau_{i+1}^{\prime}\right), x_{i+1}\left(\tau_{i+1}\right),\right. \\
\left.\left.\quad u_{i}\left(\tau_{i}\right), d_{i}\left(\tau_{i}\right), v_{i}\left(\tau_{i}^{\prime}\right), \delta_{i}\left(\tau_{i}^{\prime}\right)\right)\right]
\end{gathered}
$$

where $l: Q \times X \times U \times D \rightarrow \mathbb{R}$ is a function assigning a cost to the pieces of continuous evolution and $g: Q \times X \times$ $Q \times X \times U \times D \times \Upsilon \times \Delta \rightarrow \mathbb{R}$ is a function assigning a cost to discrete transitions. Different variants of optimal control problems can be formulated, depending on, e.g., the type of cost function, the horizon over which the optimization takes place (finite or infinite), or whether the initial and/or final states are specified.

As with continuous systems, two different approaches have been developed for addressing such optimal control problems. One is based on the maximum principle and the other on dynamic programming. Extensions of the maximum principle to hybrid systems have been proposed by numerous authors; see, for example, [11-13]. The solution of the optimal control problem with the dynamic programming approach typically requires the computation of a value function, which is characterized as a viscosity solution to a set of variational or quasi-variational inequalities $[14,15]$. This 
approach has also been extended to classes of stochastic hybrid systems; see, for example, [16,17]. Computational methods for solving the resulting variational and quasivariational inequalities are presented in [18]. For simple classes of systems (e.g., timed automata) and simple cost functions (e.g., minimum time problems) it is often possible to exactly compute the optimal cost and optimal control strategy, without resorting to numerical approximations; see, for example, [19-22].

A somewhat different optimal control problem arises when one tries to control hybrid systems using model predictive or receding horizon techniques. Generally, the aim here is to use a model to predict the future evolution of the system under different inputs and then employ optimization algorithms to select the inputs that promise the "best" future. The initial part of these inputs is applied to the system, a new measurement is taken (providing feedback), and the process is repeated. For hybrid systems, such a model predictive control approach has primarily been studied in discrete time; see, for example, [23, 24]. The toolbox of [25] provides functions for the numerical solution of hybrid model predictive control problems (and much more).

\section{Language Specification Problems}

Another type of control problem that has attracted considerable attention in the hybrid systems literature revolves around language specifications. One example of language specifications is the safety specifications. In this case a "good" set of states $W \subseteq Q \times X$ is given and the designer is asked to produce a controller that ensures that the state always stays in this set; in other words, for all runs $(\tau, q, x, v, u, \delta, d)$ of the closed-loop system

$$
\forall I_{i} \in \tau \quad \forall t \in I_{i}, \quad\left(q_{i}(t), x_{i}(t)\right) \in W .
$$

The name "safety specifications" (which is given a formal meaning in computer science) intuitively refers to the fact that such specifications can be used to encode safety requirements in a system, to ensure that nothing bad happens, e.g., in an air traffic management system to ensure that aircraft do not come closer to one another than a certain minimum distance.

Safety specifications are usually easy to meet (e.g., if aircraft never take off, mid-air collisions are impossible). To make sure that in addition to being safe the system actually does something useful, liveness specifications are usually also imposed. The simplest type of liveness specification deals with reachability: given a set of states $W \subseteq Q \times X$, design a controller such that for all runs $(\tau, q, x, v, u, \delta, d)$ of the closed-loop system

$$
\exists I_{i} \in \tau \quad \exists t \in I_{i}, \quad\left(q_{i}(t), x_{i}(t)\right) \in W .
$$

In the air traffic context a minimal liveness type requirement is to make sure that the aircraft eventually arrive at their destination. Mixing different types of specifications like the ones given above one can construct arbitrarily complex properties, e.g., ensure that the state visits a set infinitely often, ensure that it reaches a set and stays there forever after, etc. Such complex language specifications are usually encoded formally using temporal logic notation.

Controller design problems under language specifications have been studied very extensively for discrete systems in the computer science literature, mostly under the name synthesis problems. The approach was then extended to classes of hybrid systems such as timed automata (systems with continuous dynamics of the form $\dot{x}=1,[26,27]$ ) and rectangular automata (systems with continuous dynamics of the form $\dot{x} \in[l, u]$ for fixed parameters $l, u,[28])$. For systems of this type, exact and automatic computation of the controllers may be possible using model checking tools [29-31]. In all these cases the controller affects only the discrete aspects of the system evolution, i.e., the destination and timing of discrete transitions. More general language problems (e.g., where the dynamics are linear, the controller affects the continuous motion of the system) can be solved automatically in discrete time using methods from mathematical programming [25].

Extensions to general classes of hybrid systems in continuous time have been concerned primarily with computable numerical approximations of reachable sets using polyhedral approximations [32-35], ellipsoidal approximations [36], or more general classes of sets (e.g., defined using the solutions of the continuous system [37]). A useful link in this direction has been the relation between reachability problems and optimal control problems with an $l_{\infty}$ penalty function $[38,39]$. This link has allowed the development of numerical tools that use partial differential equation solvers to approximate the value function of the optimal control problems and hence indirectly characterize reachable sets [18].

\section{Concluding Remarks and Open Problems}

The topic of hybrid control has attracted considerable attention from the research community in recent years. This has produced a number of theoretical and computational methods, which are now available to the designer and have been used successfully in a wide range of applications. There are still, however, many details that need to be clarified, as well as substantial problems that have not been studied in sufficient detail. We conclude this overview by listing some of these problems (by no means an exhaustive list).

A number of interesting problems arise in the area of dynamic feedback, which is still unexplored to a large extent. The rapid development in the design of hybrid observers witnessed in recent years poses the question of how the system will perform if the state estimates that the observers produce are used in state feedback. General principles (like the separation principle in linear systems) are probably too much to hope for in a general hybrid setting, but substantial progress may still be possible for specific subclasses.

A second area that, despite numerous contributions, still poses formidable problems is the area of hybrid games. As in the robust control of continuous systems, gaming appears in hybrid systems when one adopts a non-deterministic point of view to the control of uncertain systems. Unlike continuous systems, however, even fundamental notions such as "information" and "strategy" are still the topic of debate 
in hybrid systems. It is hoped that advances in this front will eventually lead to a robust control theory for classes of uncertain hybrid systems.

Finally, stochastic hybrid systems pose a number of challenges. For example, the formulation and solution of language specifications (even of the simplest safety type) for stochastic hybrid systems is still to a large extent open. Progress in this area could come by blending results for stochastic discrete event systems with results on the $l_{\infty}$ optimal control of stochastic systems.

\section{REFERENCES}

[1] J. Lygeros, K. Johansson, S. Simić, J. Zhang, and S. Sastry, "Dynamical properties of hybrid automata," IEEE Transactions on Automatic Control, vol. 48, no. 1, pp. 2-17, January 2003.

[2] D. Liberzon and A. Morse, "Basic problems in stability and design of switched systems," IEEE Control Systems Magazine, vol. 19, pp. 59-70, October 1999.

[3] R. De Carlo, M. Branicky, S. Pettersson, and B. Lennarston, "Perspectives and results on the stability and stabilizability of hybrid systems," Proceedings of the IEEE, vol. 88, no. 7, pp. 1069-1082, July 2000.

[4] M. Wicks, P. Peleties, and R. De Carlo, "Switched controller synthesis for the quadratic stabilization of a pair of unstable linear systems," European Journal of Control, vol. 4, pp. 140-147, 1998.

[5] A. V. Savkin, E. Skafidas, and R. Evans, "Robust output feedback stabilizability via controller switching," Automatica, vol. 35, no. 1, pp. 69-74, 1999.

[6] J. Hespanha and A. Morse, "Switching between stabilizing controllers," Automatica, vol. 38, no. 11, pp. 1905-1917, November 2002.

[7] X. Xu and G. Zhai, "On practical stability and stabilization of hybrid and switched systems," in Hybrid Systems Computation and Control, ser. LNCS, R. Alur and G. Pappas, Eds. Berlin: Springer-Verlag, 2004, no. 2993, pp. 615-630.

[8] X. Xu and P. Antsaklis, "Stabilization of second order LTI switched systems," International Journal of Control, vol. 73, no. 14, pp. 12611279, September 2000.

[9] Y. Gao, J. Lygeros, M. Quincampoix, and N. Seube, "On the control of uncertain impulsive systems: Approximate stabilization and controlled invariance," International Journal of Control, 2005, to appear.

[10] C. Yuan and J. Lygeros, "Stabilization of a class of stochastic systems with Markovian switching," in Mathematical Theory of Networks and Systems (MTNS04), Leuven, Belgium 2004.

[11] G. Grammel, "Maximum principle for a hybrid system via singular pertrubations," SIAM Journal of Control and Optimization, vol. 37, no. 4, pp. 1162-1175, 1999.

[12] H. J. Sussmann, "A maximum principle for hybrid optimal control problems," in IEEE Conference on Decision and Control, Phoenix, Arizona, December 7-10, 1999, pp. 425-430.

[13] M. Shaikh and P. Caines, "On the optimal control of hybrid systems: optimal trajectories, switching times and location schedules," in $\mathrm{Hy}$ brid Systems: Computation and Control, ser. LNCS, O. Maler and A. Pnueli, Eds. Berlin: Springer-Verlag, 2003, no. 2623, pp. 466481.

[14] A. Bensoussan and J. Menaldi, "Hybrid control and dynamic programming," Dynamics of Continuous, Discrete and Impulsive Systems, no. 3, pp. 395-442, 1997.

[15] M. Branicky, V. Borkar, and S. Mitter, "A unified framework for hybrid control: Model and optimal control theory," IEEE Transactions on Automatic Control, vol. 43, no. 1, pp. 31-45, 1998.

[16] M. Ghosh, A. Arapostathis, and S. Marcus, "Optimal control of switching diffusions with application to flexible manufacturing systems," SIAM Journal on Control Optimization, vol. 31, no. 5, pp. 1183-1204, September 1993.

[17] A. Bensoussan and J. Menaldi, "Stochastic hybrid control," Journal of Mathematical Analysis and Applications, vol. 249, pp. 261-288, 2000.

[18] I. Mitchell, A. Bayen, and C. Tomlin, "Validating a HamiltonJacobi approximation to hybrid system reachable sets," in Hybrid Systems: Computation and Control, ser. LNCS, M. Di Benedetto and A. Sangiovanni-Vincentelli, Eds. Berlin: Springer-Verlag, 2001, no. 2034, pp. 418-432.
[19] E. Asarin and O. Maler, "As soon as possible: Time optimal control for timed automata," in Hybrid Systems: Computation and Control, ser. LNCS, F. W. Vaandrager and J. H. van Schuppen, Eds. Berlin: Springer-Verlag, 1999, no. 1569, pp. 19-30.

[20] R. Alur, S. La Torre, and G. Pappas, "Optimal paths in weighted timed automata," in Hybrid Systems: Computation and Control, ser. LNCS, M. Di Benedetto and A. Sangiovanni-Vincentelli, Eds. Berlin: Springer-Verlag, 2001, no. 2034, pp. 49-62.

[21] G. Berhmann, , A. Fehnker, T. Hune, K. Larsen, P. Pettersson, J. Romijn, and F. Vaandrager, "Minimum cost reachability for priced timed automata," in Hybrid Systems: Computation and Control, ser. LNCS, M. Di Benedetto and A. Sangiovanni-Vincentelli, Eds. Berlin: Springer-Verlag, 2001, no. 2034, pp. 147-161.

[22] O. Maler, "On optimal and suboptimal control in the presence of adversaries," in Proceedings of WODES04, 2004, pp. 1-12.

[23] A. Bemporad and M. Morari, "Control of systems integrating logic dynamics and constraints," Automatica, vol. 35, no. 3, pp. 407-427, March 1999.

[24] D. Mayne and S. Rakovic, "Model predictive control of contrained, piecewise affine, discrete time systems," International Journal of Robust and Nonlinear Control, vol. 13, no. 3, pp. 261-279, 2003.

[25] M. Kvasnica, P. Grieder, M. Baotic, and M. Morari, "The multiparametric toolbox (MPT)," in Hybrid Systems Computation and Control, ser. LNCS, R. Alur and G. Pappas, Eds. Berlin: SpringerVerlag, 2004, no. 2993, pp. 448-462.

[26] E. Asarin, O. Maler, and A. Pnueli, "Symbolic controller synthesis for discrete and timed systems," in Proceedings of Hybrid Systems II, ser. LNCS, P. Antsaklis, W. Kohn, A. Nerode, and S. Sastry, Eds. Berlin: Springer-Verlag, 1995, no. 999, pp. 1-20.

[27] M. Heymann, F. Lin, and G. Meyer, "Synthesis and viability of minimally interventive legal controllers for hybrid systems," Discrete Event Dynamic Systems: Theory and Applications, vol. 8, no. 2, pp. 105-135, June 1998.

[28] H. Wong-Toi, "The synthesis of controllers for linear hybrid automata," in IEEE Conference on Decision and Control, San Diego, California, December 10-12 1997, pp. 4607-4613.

[29] J. Bengtsson, K. Larsen, F. Larsson, P. Petterson, and W. Yi, "UPAAL: A tool suit for automatic verification of real-time systems," in Hybrid Systems III, ser. LNCS. Berlin: Springer-Verlag, 1996, no. 1066, pp. 232-243.

[30] C. Daws, A. Olivero, S. Trypakis, and S. Yovine, "The tool KRONOS," in Hybrid Systems III, ser. LNCS, R. Alur, T. Henzinger, and E. Sontag, Eds. Berlin: Springer-Verlag, 1996, no. 1066, pp. 208-219.

[31] T. A. Henzinger, P. H. Ho, and H. W. Toi, "A user guide to HYTECH," in TACAS 95: Tools and Algorithms for the Construction and Analysis of Systems, ser. LNCS, E. Brinksma, W. Cleaveland, K. Larsen, T. Margaria, and B. Steffen, Eds., no. 1019. Berlin: Springer-Verlag, 1995, pp. 41-71.

[32] M. Greenstreet and I. Mitchell, "Integrating projections," in Hybrid Systems: Computation and Control, ser. LNCS, S. Sastry and T. Henzinger, Eds. Berlin: Springer-Verlag, 1998, no. 1386, pp. 159-174.

[33] A. Chutinam and B. Krogh, "Verification of polyhedral-invariant hybrid automata using polygonal flow pipe approximations," in Hybrid Systems: Computation and Control, ser. LNCS, F. W. Vaandrager and J. H. van Schuppen, Eds. Berlin: Springer-Verlag, 1999, no. 1569, pp. 76-90.

[34] E. Asarin, O. Bournez, T. Dang, O. Maler, and A. Pnueli, "Effective synthesis of switching controllers for linear systems," Proceedings of the IEEE, vol. 88, no. 7, pp. 1011-1025, July 2000.

[35] P. Saint-Pierre, "Approximation of viability kernels and capture basins for hybrid systems," in European Control Conference, Porto, September 4-7, 2001, pp. 2776-2783.

[36] O. Botchkarev and S. Tripakis, "Verification of hybrid systems with linear differential inclusions using ellipsoidal approximations," in Hybrid Systems: Computation and Control, ser. LNCS, N. Lynch and B. H. Krogh, Eds. Berlin: Springer-Verlag, 2000, no. 1790, pp. 73-88.

[37] X. Koutsoukos, P. Antsaklis, J. Stiver, and M. Lemmon, "Supervisory control of hybrid systems," Proceedings of the IEEE, vol. 88, no. 7, pp. 1026-1049, July 2000.

[38] C. Tomlin, J. Lygeros, and S. Sastry, "A game theoretic approach to controller design for hybrid systems," Proceedings of the IEEE, vol. 88, no. 7, pp. 949-969, July 2000.

[39] J. Lygeros, "On reachability and minimum cost optimal control," Automatica, vol. 40, no. 6, pp. 917-927, 2004. 